\title{
Oscillatory Behaviors of Second Order Forced Functional Differential Equation.
}

\author{
Birma John Javan, Egurube Michael O. And Wangercha Wilson Adams. \\ Department of Mathematics, School of Pure and Applied Sciences, Modibbo Adama University of Technology \\ Yola, Nigeria.
}

\begin{abstract}
Oscillatory behaviors of second order forced functional differential equation is considered. The oscillation of this equation is shown to be maintained under the effect of certain forcing terms, and the oscillatory equation can serve as mathematical tool for simulation of processes and phenomina observed in control theory.
\end{abstract}

Key words: Amplitude, functional differential equations, Damped oscillation, Oscillatory behaviors.

\section{Introduction}

Oscillatory behaviors describe the behaviors of solutions of differential equations, while oscillation is a regular movement of the solutions between one position and another.

Forced oscillations are obtained if we let an external force act on the body or if we simply add a new force to the existing force. The oscillation of a real valued function on an interval is the difference between its least upper bound and greatest lower bound there, the oscillation of a real valued function at a point $\mathrm{x}$ is the limit of the oscillation of the function on the interval $[x-e, x+e]$ as $\mathrm{e} \rightarrow 0$

Functional differential equations of the form

$x^{\prime \prime}+c x^{\prime}+\omega_{0} x=0$

Where $\omega_{0}=\sqrt{k / m}$ is the undamped oscillation frequency. (1) Shall be referred to as the damped oscillator equation. Its solution is called oscillatory if it has arbitrarily large number of zeros, otherwise the solution is said to be non oscillatory. An equation is said to be oscillatory if all of its solutions are oscillatory [5] The solution of an equation is called oscillatory if the sets of its zeros are unbounded from above, otherwise it is said to be nonoscillatory, and the equation is said to be oscillatory when all the solutions are oscillatory [4] The major problem in the theory of oscillation is the problem of maintaining oscillations under the effect of a forcing term. This study is therefore to investigate the effect of a forcing term on the unforced equation, and to investigate the criteria for selecting appropriate forcing terms.

\section{Role of Oscillatory Differential Equations}

Oscillatory functional differential equations can be used as mathematical tools for simulation of process and phenomena observed in control theory, most especially the second order forced functional differential equations which can govern the simulation of process in control theory.

In industrial quality control the idea of testing have always been adapted and extended in various ways to serve basic practical needs. The control chart for the sample mean of testing made, shows the lower control limit (LCL), the central control limit (CCL) and the upper control limit (UCL) (Kreyszig1999). This clearly obeys the forced oscillatory equations. The two control limits lower control limit and the upper control limit corresponds to the greatest lower bound, the least upper bound of the oscillation, as soon as the sample mean falls outside the range between the two control limit we reject the hypothesis and asserts that the production is out of control.

\section{Definition of Basic Terms}

Oscillation; oscillation is any effect the varies periodically back and forth between two values [6]

Functional differential equations: a functional differential equation is an equation in which the derivatives $y^{(n)}(t)$ of an unknown function $y$ has a value at t. [1]

Damped oscillation is the linear motion of a particle subject both to an elastic restoring force proportional to its displacement and to a frictional force in the direction opposite to its motion and proportional to its speed or any oscillation in which the amplitude of the oscillation quantity decreases with time.

Sinusoidal function: The real or complex function $\sin (\mathrm{u})$ or any function with analogous continuous periodic behaviors. 
Many investigations and studies have been carried out by authors on theory of oscillations, but the problem of maintaining oscillation under the effect of a forcing term has always been left out. The oscillations of all solutions of unforced functional differential equations

$$
x^{\prime \prime}+c x^{\prime}+\omega_{0} x=0
$$

Whose solution is of the form

$x(t)=a e^{-\lambda t} \cos \left(\omega_{1} t-\sigma\right)$

Where $a \in C\left[t_{0}, \infty\right), \lambda>0, \sigma>0, x \neq 0$

Is not generally maintained if one considers the forced equations by adding a forcing term to the right hand side of equation (1.2).

[2] Studied oscillation theory for functional differential equation

$$
x^{\prime}(t)+q(t) x(\phi(t))=0, t \geq t_{0}
$$

Where $q \in C\left(\left[t_{0}, \infty\right), \mathfrak{R}^{+}, 0\right), \phi \in C\left[t_{0}, \infty\right), \phi(t)<t$,

And obtained the following results;

That every solution of equation (1.3) oscillates if

$\lim _{t \rightarrow \infty} \operatorname{Inf} \int_{\sigma}^{t} q(s) d s>\frac{1}{e}$.

[8] Investigated oscillations for first order linear differential equation

$$
x^{\prime}(t)+q(t)\left[x(\phi(t) 0]=0, t>t_{0}\right.
$$

Where $q \in C\left[t_{0}, \infty\right), \phi(t)<t, \infty \in(1, \infty)$,

And obtained the oscillatory behavior, that if $\phi$ be continuously differentiable, further, suppose that there exists a continuous differentiable function $\varphi$ such that $\varphi(t)>0$ and $\lim _{t \rightarrow \infty} \varphi(t)=\infty$,

$$
\operatorname{Lim}_{t \rightarrow \infty} \operatorname{Sup} \frac{\alpha \varphi^{\prime}(\phi(t) \phi(t)}{\varphi^{\prime}(t)}<1, \operatorname{andLim}_{t \rightarrow \infty} \operatorname{Im} f \frac{q(t) e^{-\varphi(t)}}{\varphi^{\prime}(t)}>0
$$

Then every solution of the linear equation (1.4) oscillates.

[1] Studied equation of the type

$$
\begin{aligned}
& x^{\prime}(t)+q(t) x^{a}(t-\phi)=0 \\
& x^{\prime \prime}(t)+q(t) g\left(x(t-\phi)^{\prime}=0\right.
\end{aligned}
$$

Where $q \in C([t, \infty),(0, \infty)], \phi, \tau \in(0, \infty), g: \mathfrak{R} \rightarrow \mathfrak{R}$ such that $x g(x)>0$ for $x \neq 0$, g is nondecreasing.

Necessary and sufficient conditions in terms of coefficient were obtained for all solutions of the prescribed equations which were oscillatory.

[5] Studied the oscillation of the equation

$$
x^{(n)}(t)+p(t) x(t-\tau)=0, t \geq t_{0}
$$

$t \neq t_{k}, \tau>0,0 \leq t_{0}<t_{1}<t_{2}<\ldots<t_{k}$ Such that $t_{k} \rightarrow \infty$ as $k \rightarrow \infty$, and established oscillatory results based on combinations of the following conditions:

(i) $\operatorname{Lim}_{t \rightarrow \infty} \int_{t_{0}}^{t} \pi_{t_{0}}<t_{k}<S \frac{a_{k}^{i}}{a_{k}^{(i-1)}} d s=\infty, i=1,2, \ldots n-1$.

(ii) There exist a positive integer $k_{0}$ such that for $k \geq k_{0}$ and for any natural number $n \in\{1,3, \ldots n-1\} a_{k}^{i}>C_{k}^{i},(i=0,1,2, \ldots i-1)$.

While the above investigations concerns unforced functional differential equations, we are motivated to investigate the problems of maintaining oscillations under the effect of a forcing term.

\section{Forced Oscillations of Linear Equations}

Forced motions are obtained, if an external force acts on the body, to gate this one simply add a new force to those forces (equation (1.2)) this gives the nonhomogeneous differential equation

$$
x^{\prime \prime}+c x^{\prime}+\omega_{0}^{2} x=\omega_{0}^{2} X_{0}
$$


A corresponding solution is a response of the system to the driving force of a particular interest, is the periodic force which may be considered as sinusoidal force say

$x(t)=x_{0} \cos (\omega t)$

If $\mathrm{c}=0$ (2.1) is undamped and the solution $x_{p}(t)$ becomes larger and larger this means that a system with very small damping undergoes large vibrations that can destroy the system.

When $\omega$ is close to $\omega_{0}$ a type of oscillation that grows large and larger until it riches the upper limit and then begins to come back to the origin, is obtained. This is what happens in forced undamped oscillations when the difference of the input and the natural frequencies are so small.

\section{Damped Forced Oscillation}

In free oscillation energy conserving physical system which exhibits harmonic oscillation about a stable equilibrium state is encountered, the main feature of such oscillation is that once excited it never dies away. [7]

However, majority of the oscillation system that one encounters in every day life suffer some sorts of irreversible energy loss whilst they are in motion, which is due for instance to frictional or viscose heat generations. We would therefore expect oscillations excited in such a system eventually be damped away.

If there is damping then $\mathrm{c}>0$, the general solution $x_{h}$ of the homogeneous equation (1.2) is $x_{h}(t)=e^{-\alpha t}\left(A \cos \omega^{*} t+B \cos \omega^{*} t\right)$. this solution approaches zero as $t$ goes to infinity. Now the general solution of the nonhomogeneous equation (2.1) is $x=x_{p}+x_{h}$. it approaches the steady state solution which leads to a harmonic oscillation whose frequency is that of the input, because no physical system is completely undamped.

\section{Driven Oscillation}

When a damped mechanical oscillator is set into motion the oscillation dies away due to frictional energy losses, infarct the only way of maintaining the amplitude of a damped oscillator equation is to continuously feed energy into the system in such a manner as to off set the frictional losses. A steady (constant amplitude) oscillation of this type is called Driven oscillation. We would generally expect the periodically driven oscillator to eventually settle down to a steady pattern of oscillation, with the same frequency in which the frictional energy loss per cycle is exactly matched by the work done per cycle. The equation of motion of the system is

$$
x^{\prime \prime}+c x^{\prime}+\omega_{0}^{2} x=\omega_{0} X
$$

Where $c>0$, is a damping constant and $\omega_{0}>0$ the damped oscillation frequency. The time evolution equation of the system takes the form

$$
x^{\prime \prime}+c x^{\prime}+\omega_{0}^{2} x=\omega_{0}^{2} X_{0} \cos (\omega t)
$$

We now search for the solution of (3.2) in the form

$$
x(t)=x_{0} \cos (\omega t-\sigma)
$$

Here $x_{0}>0$ is the amplitude of the driven oscillation while $\sigma$ is the phase lag of the oscillation. Differentiating (3.3) twice we have

$$
\begin{aligned}
& x^{\prime}=-\omega x_{0} \sin (\omega t-\sigma) \\
& x^{\prime \prime}=-\omega^{2} x_{0} \cos (\omega t-\sigma)
\end{aligned}
$$

Equation (3.5) becomes

$$
\begin{gathered}
\left(\omega_{0}^{2}-\omega^{2}\right) x_{0} \cos (\omega t-\sigma)-c \omega x_{0} \sin (\omega t-\sigma)=\omega_{0}^{2} X_{0} \cos (\omega t) \\
{\left[x_{0}\left(\omega_{0}^{2}-\omega^{2}\right) \cos \sigma+x_{0} c \omega \sin \sigma-\omega_{0}^{2} X_{0}\right] \cos (\omega t)+} \\
{\left[x_{0}\left(\omega_{0}^{2}-\omega^{2}\right) \sin \sigma-c \omega \cos \sigma\right] \sin (\omega t)=0}
\end{gathered}
$$

For equation (3.7) to be satisfied the coefficients of $\cos (\omega t)$ and $\sin (\omega t)$ most separately equate to zero,

$x_{0}\left(\omega_{0}^{2}-\omega^{2}\right) \cos \sigma+x_{0} c \omega \sin \sigma-\omega_{0}^{2} X_{0}=0$

$\left(\omega_{0}^{2}-\omega^{2}\right) \sin \sigma-c \omega \cos \sigma=0$

If we combine (3.7) and (3.8) we gate 


$$
\begin{aligned}
& x_{0}=\frac{\omega_{0}^{2} X_{0}}{\left[\left(\omega_{0}^{2}-\omega^{2}\right)^{2}+c^{2} \omega^{2}\right]^{1 / 2}} \\
& \sigma=\tan ^{-1}\left(\frac{c \omega}{\omega_{0}^{2}-\omega^{2}}\right)
\end{aligned}
$$

Because (3.8) gives

$$
\begin{aligned}
& \tan \sigma=\frac{c \omega}{\omega_{0}^{2}-\omega^{2}} \\
& \cos \sigma=\frac{\omega_{0}^{2}-\omega^{2}}{\left[\left(\omega_{0}^{2}-\omega^{2}\right)^{2}+c^{2} \omega^{2}\right]^{1 / 2}} \\
& \sin \sigma=\frac{c \omega}{\left[\left(\omega_{0}^{2}-\omega^{2}\right)^{2}+c^{2} \omega^{2}\right]^{1 / 2}}
\end{aligned}
$$

Investigating the dependence of the amplitude $x_{0}$ and the phase lag $\sigma$ of the driven oscillation on the driving frequency $\omega, \frac{x_{0}}{X_{0}}$ and $\sigma$ as a function of $\omega$ for various different values of $\frac{c}{\omega_{0}}=1 / Q$, this will give us quality factor $\mathrm{Q}$. it can be observed that as the amount of damping in the system is decreased the amplitude of the response riches its peak at the natural frequency of the oscillation of the system, so a weakly damped oscillator can be driven to large amplitude by the application of a relatively small amplitude external driving force which oscillates at a frequency close to the resonant frequency. The response of the oscillator is in phase (i.e. $\sigma \approx 0$ ) with the external drive for driving frequencies well bellow the resonant frequency, is in phase quadrature (i.e. $\sigma=\frac{\pi}{2}$ ) at the resonant frequency, and is in anti-phase (i.e. $\sigma \approx \pi$ ) for frequency well above the resonant frequency.

When the driving frequency matches the resonant frequency the ratio of the amplitude of the driven oscillation is the quality factor $\mathrm{Q}$, which can be regarded as the resonant amplification factor.

\section{Response of Transient oscillator}

If we consider the time evolution equation of damped oscillation

$$
x^{\prime \prime}+c x^{\prime}+\omega_{0}^{2} x=\omega_{0}^{2} X_{0} \cos (\omega t)
$$

The steady state solution to this equation is of the form

$$
x_{p}(t)=x_{0} \cos (\omega t-\sigma)
$$

Where

$$
\begin{aligned}
& x_{0}=\frac{\omega_{0}^{2} X_{0}}{\left[\left(\omega_{0}^{2}-\omega^{2}\right)^{2}+c^{2} \omega^{2}\right]^{1 / 2}} \\
& \sigma=\tan ^{-1}\left(\frac{c \omega}{\omega_{0}^{2}-\omega^{2}}\right)
\end{aligned}
$$

The general solution of (3.14) should contain two arbitrary constants. However (3.15) contains no arbitrary constants, if we add any solution of (1) to equation (3.15) then the result will still be a solution to equation (3.14). The solution to equation (3.14) can be written as

$$
x_{h}(t)=A e^{-c t / 2} \cos \left(\omega_{1} t\right)+B e^{-c t / 2} \sin \left(\omega_{1} t\right)
$$

Where $\omega_{1}=\left(\omega_{0}^{2}-c^{2} / 4\right)^{1 / 2}$, A and B are arbitrary constants. So that a more general solution to (3.14) is

$$
\begin{aligned}
& x(t)=x_{p}(t)+x_{h}(t) \\
& x(t)=x_{0} \cos (\omega t-\sigma)+A e^{-c t / 2} \cos \left(\omega_{1} t\right)+B e^{-c t / 2} \sin \left(\omega_{1} t\right)
\end{aligned}
$$

The constants A and B are determined by the initial conditions, the general solution to (3.14) consist of two parts, first the solution (3.15) which oscillates at the driving frequency $\omega$ with a constant amplitude and 
which is independent of the initial conditions, second the solution (3.18) which oscillates at natural frequency $\omega_{1}$ with an amplitude which decays exponentially in time, and which depends on initial conditions.

The former is termed the time asymptotic so0lution since if we wait long enough then it becomes dominant; the later is called the transient solution, since if we wait long enough it decays away.

Suppose that the system is in its equilibrium that is $x(0)=x^{\prime}(0)$ it follows from (3.20) that

$$
\begin{aligned}
& x(0)=x_{0} \cos \sigma+A=0 \\
& x^{\prime}(0)=x_{0} \omega \sin \sigma-c / 2 A+B \omega_{1}=0
\end{aligned}
$$

These equations can be solved to give

$$
A=-x_{0} \cos \sigma
$$

$$
B=-x_{0}\left[\frac{\omega \sin \sigma+(c / 2) \cos \sigma}{\omega_{1}}\right]
$$

For driving frequency close to the resonant frequency we can write

$$
\begin{aligned}
& x_{0}=\frac{X_{0} \omega_{0}}{\left[4\left(\omega_{0}-\omega\right)^{2}+c^{2}\right]^{1 / 2}} \\
& \cos \sigma \approx \frac{2\left(\omega_{0}-\omega\right)}{\left[4\left(\omega_{0}-\omega\right)^{2}\right]^{1 / 2}} \\
& \sin \sigma \approx \frac{c}{\left[4\left(\omega_{0}-\omega\right)^{2}+c^{2}\right]^{1 / 2}}
\end{aligned}
$$

The solution (3.20) combined with (3.23) to (3.27) reduces to

$$
\begin{aligned}
& x(t)=X_{0}\left[\frac{2 \omega_{0}\left(\omega_{0}-\omega\right)}{4\left(\omega_{0}-\omega\right)^{2}+c^{2}}\right]\left[\cos (\omega t)-e^{-c t / 2} \cos \left(\omega_{0} t\right)\right]+ \\
& X_{0}\left[\frac{\omega_{0} c}{4\left(\omega_{0}-\omega\right)^{2}+c^{2}}\right]\left[\sin (\omega t)-e^{-c t / 2} \sin \left(\omega_{0} t\right)\right]
\end{aligned}
$$

If the driving frequency is equal to the resonant frequency that is

$\omega=\omega_{0}$, in this case equation (3.28) reduces to

$x(t)=X_{0} Q\left(1-e^{-c t / 2}\right) \sin (\omega t)$

$Q=\frac{\omega_{0}}{c}$. Thus the driving response oscillates at the resonant frequency $\omega_{0}$ since both the time asymptotic and the transient solution oscillates at this frequency.

However the amplitude of the oscillation grows monotonically as

$$
a(t)=X_{0} Q\left(1-e^{-c t / 2}\right)
$$

And so takes a time of order $\mathrm{c}^{-1}$ to attain its final value $X_{0} Q$, which is larger than the driving amplitude by resonant amplification factor. In absence of damping force that is when $\mathrm{c}=0$ equation (3.28) yields

$$
x(t)=X_{0}\left(\frac{\omega_{0}}{\omega_{0}-\omega}\right) \sin \left[\left(\omega_{0}-\omega\right) t / 2\right] \sin \left[\left(\omega_{0}+\omega\right) t / 2\right]
$$

Using the trigonometric identity

$$
\cos a-\cos b \equiv-2 \sin [(a+b) / 2] \sin [(a-b) / 2]
$$

The driven response oscillates relatively rapidly at the sum frequency $\left(\omega_{0}+\omega\right) / 2$ with amplitude $a(t)=X \omega_{0}\left[\omega_{0} /\left(\omega_{0}-\omega\right)\right] \sin \left[\left(\omega_{0}-\omega\right) / t\right]$

This modulates relatively slowly at the difference frequency $\left(\omega_{0}-\omega\right) / 2$. 
(Assuming that $\omega$ is close to $\left.\omega_{0}\right), T_{0}=\frac{2 \pi}{\omega_{0}} \quad$ and the amplitude modulations are called beats, and are produced whenever two sinusoidal oscillations of similar amplitude and slightly different frequency are superposed. In this case the oscillations are the time asymptotic solutions, which oscillates at the driving frequency $\omega$, and the transient solution which oscillates at the resonant frequency $\omega_{0}$, the beats modulates at the difference frequency $\left(\omega_{0}-\omega\right) / 2$ in the limit $\omega \rightarrow \omega_{0}$, equation (3.30) yields

$x(t)=\frac{X_{0}}{2} \omega_{0} t \sin \left(\omega_{0} t\right)$

Since $\sin x \approx x$ when $|x|<1$ thus the resonant response of a driven undamped oscillator is an oscillation at the resonant frequency whose amplitude $a(t)=\left(X_{0} / 2\right) \omega_{0} t$ increases linearly in time. The nonresonant response of a driven damped oscillator obtained from equation (3.28) where $T_{0}=\frac{2 \pi}{\omega_{0}}$ shows that the driven response grows, but eventually settles down to a steady pattern of oscillation.

\section{Conclusion}

In conclusion it is evident that the oscillation of the unforced equation is maintained under the effect of certain forcing terms.

If a periodic (sinusoidal) forcing term is added at angular frequency $\omega$, the same solution regimes are again obtained. The resulting motion is still periodic (after an initial transient response corresponding to the solution of the unforced case has died out), but it has an amplitude different from the forcing amplitude.

The only way of maintaining the amplitude of a damped oscillator in a physical system is to continuously feed energy into the system in such a manner as to offset the frictional losses.

\section{Reference}

[1] Dass P. and Misra N. (1997) Necessary and sufficient conditions for the solution of a functional differential equations. To be oscillatory. Journal of mathematical analysis and applications.

[2] Erbe L. H. and Qingkai K. H. (1995) oscillation theory for functional differential equations. Volume 190 of monographs and text books in pure and applied mathematics. Marcel Decker Inc.New York.

[3] Hamadani G.G. (1995). Oscillatory behavior of $\mathrm{n}^{\text {th }}$ order forced functional differential equations. Journal of mathematical analysis and applications.

[4] Kreyszig E. (1999). Advanced engineering mathematics $8^{\text {th }}$ edition.

[5] Lijun P. and Jinde C. (2010) Oscillation of even order linear implosive delay differential equations. Journal of differential equations and applications. 2(2) 163-176.

[6] McGrew-Hill (1978). Dictionary of science and mathematics.

[7] Richard F. (2010) Simple harmonic oscillator equation. From Math World-a wolfram web resource. http/mathworld.wolfram.com. applications.

[8] Tang X.H. (2002). Oscillation for first order supper linear delay differential equations. Journal, London mathematical society. 